ÉDITORIAL

\section{La recherche sur le lieu et la santé au Canada}

\author{
Louise Potvin, Ph.D. ${ }^{1}$ \\ Michael V. Hayes, Ph.D. ${ }^{2}$
}

$\mathrm{D}$ epuis les 30 dernières années au Canada, le discours sur les politiques sanitaires insiste sur un double objectif : améliorer l'état de santé global de la population tout en réduisant l'ampleur des inégalités en santé associées au statut socioéconomique, à l'appartenance ethnique, au sexe ou à la géographie. Les responsables de la planification et de la mise en oeuvre des politiques et des programmes de santé publique se préoccupent vivement de connaître la configuration géographique des déterminants de la santé et des résultats sanitaires et ses raisons, d'une part parce que la tendance actuelle est à la décentralisation et à la régionalisation des systèmes de santé provinciaux au Canada, et de l'autre, parce que l'on s'intéresse de plus en plus aux stratégies locales et aux approches territoriales pour l'élaboration des programmes de santé publique (voir Potvin et coll. ${ }^{1}$ ). Par ailleurs, avec l'évolution constante des systèmes d'information géographique et leur utilisation accrue dans le secteur de la santé, l'intérêt des chercheurs se porte de plus en plus sur les relations entre la santé des gens et les caractéristiques des lieux de la vie quotidienne. Les déterminants sociaux de la santé sont imprégnés des aspects de la géographie sociale et physique (généralement implicites) qui nous touchent au cours de la vie. L'un des grands objectifs de la recherche sur le lieu et la santé demeure de rendre ces caractéristiques explicites.

Comme beaucoup d'autres grands domaines d'étude en santé publique et des populations, la recherche sur le lieu et la santé se présente principalement sous l'aspect d'un ensemble d'observations empiriques en quête d'une explication générale. La liste des déterminants de la santé et des indicateurs de résultats qui présentent une configuration spatiale et qui sont associés aux caractéristiques contextuelles du lieu ne cesse de s'allonger. Les possibilités de déceler des configurations géographiques dans les phénomènes de santé semblent infinies, comme on le voit dans le nombre croissant d'aspects du lieu dont on étudie les associations avec la santé. L'élaboration, au cours des 20 dernières années, d'algorithmes de calcul d'intervalles de confiance autour d'estimations de coefficients de régression hiérarchique, ainsi que la mise au point de logiciels de modélisation hiérarchique conviviaux, ont fait exploser la recherche sur le lieu et la santé. Deux grandes revues scientifiques internationales (Health \& Place et Journal of Epidemiology and Community Health) publient même principalement des rapports de recherche analysant de telles configurations géographiques. La signification précise de tous ces liens nous échappe encore, cependant.

D'un point de vue analytique, la notion du « lieu et de la santé " présente une énigme fondamentale. D'une part, nous subissons tous les influences diverses et simultanées du « lieu » sur notre santé par l'intermédiaire de nos relations quotidiennes (avec nos intimes, nos voisins, nos collègues et nos connaissances); du contexte social de ces rencontres et de la signification que nous leur donnons; de notre exposition physique simultanée à l'air que nous respirons et aux choses que nous mangeons, absorbons ou assimilons autrement; et des ressources (documentaires et non documentaires) auxquelles nous avons accès dans le contexte socio-spatial de notre vie. Toute personne vivante subit ces influences. C'est là l'essence de ce que Nancy Krieger appelle le modèle " écosocial » de la santé2. Par contre, notre capacité de mesurer empiriquement ces influences est très limitée par les sources de données, statiques et transversales (comme le Recensement), qui relient les attributs indivi-

1. Département de médecine sociale et préventive, Université de Montréal; Centre de recherche Léa-Roback sur les inégalités sociales de santé de Montréal, Université de Montréal; Groupe de recherche interdisciplinaire en santé, Université de Montréal, Montréal (Québec)

2. Faculté des sciences de la santé de l'Université Simon Fraser, Burnaby (Colombie-Britannique) duels à des lieux précis, mais n'ont pas les qualités dynamiques et relationnelles des phénomènes que nous cherchons à comprendre. La notion de lieu (les espaces de la vie quotidienne auxquels nous attribuons un sens) est vague du point de vue théorique, et en général, elle est opérationnalisée empiriquement comme un contenant spatial défini par les unités administratives de données disponibles (quartier, secteur de recensement, petite région, zone de planification, code postal). Il est pris pour acquis que les résidents de ces unités y passent toute ou une grande partie de leur vie. De toute évidence, les chercheurs sont limités par la complexité et l'envergure des relations dynamiques de la vie quotidienne, sans parler de la disponibilité des données.

Plusieurs enjeux soulevés par les études empiriques auraient besoin d'être développés sur le plan théorique. La façon dont on conçoit l'espace et le lieu est une question omniprésente dans les études publiées sur le lieu et la santé. Au-delà de l'opposition entre les effets compositionnels et contextuels, les théories échafaudées ne parviennent toujours pas à déterminer les éléments contextuels liés au lieu qui sont en cause dans la configuration spatiale de la santé, à l'exception remarquable de la notion de "structure de possibilités " de Macintyre et Ellaway ${ }^{3}$. Une telle structure comprend cinq aspects du quartier : (1) les éléments physiques partagés par tous les résidents, comme la qualité de l'air ou la présence de produits toxiques; (2) la présence de milieux (à la maison, au travail et dans les loisirs) propices aux modes de vie sains; (3) des services de haute qualité pour tous les segments de la population, y compris des écoles, des bibliothèques, des moyens de transport et autres services de proximité; (4) les caractéristiques socioculturelles qui sont le produit de l'histoire et de l'évolution du quartier, et qui en forment le tissu social; et (5) la réputation du secteur, déterminée par les observations des résidents eux-mêmes et des autorités compétentes. La notion de structure de possibilités englobe des réalités beaucoup plus vastes que les biens physiques et matériels, mais une grande partie de la recherche sur le lieu et la santé traite encore les ressources matérielles comme des corrélats de la santé.

Un deuxième enjeu, plus général, a trait à l'opposition "structures/activité 
humaine " en sciences sociales et pourrait s'exprimer sous la forme d'une interrogation sur les interactions croisées entre le lieu et les gens ${ }^{4}$. À vrai dire, comme la plupart des articles de recherche sur le lieu et la santé reposent sur des études transversales, il est très difficile d'examiner l'action réciproque des caractéristiques des lieux et des gens. La structure des fichiers et l'absence de données longitudinales sur les caractéristiques des lieux amènent les chercheurs à énoncer que les structures ont préséance sur l'activité humaine. Mais la relation est sûrement réciproque, et c'est cette réciprocité qui reste encore à étudier.

Un troisième enjeu a trait à l'échelle à laquelle des liens peuvent exister entre le lieu et la santé. Voilà plus de 150 ans que l'on observe des gradients sociaux dans les résultats sanitaires de différentes classes sociales; de tels gradients ont été trouvés pratiquement partout où on les a cherchés. Étant donné l'effet des forces du marché sur la configuration socio-spatiale des peuplements, on trouve souvent dans les petites régions des concentrations de personnes au profil socioéconomique semblable. À quelle échelle ou à quelles échelles pouvons-nous déceler des inégalités réelles et significatives dans l'état de santé?

En plus de ces enjeux théoriques, il faut, sur le plan pratique, déterminer l'importance ou l'utilité de la recherche sur le lieu et la santé pour les décideurs. Lors d'un atelier sur ce sujet coparrainé par l'Initiative sur la santé de la population canadienne (ISPC) et le Centre LéaRoback en avril 2005, après avoir écouté les exposés de chercheurs financés par l'ISPC, des décideurs ont souligné qu'ils avaient surtout besoin d'informations pratiques, plus utiles que bon nombre des enjeux théoriques dont il est question dans les travaux de recherche ${ }^{5}$. Les décideurs apprécient beaucoup les recommandations concrètes, mais la communauté de recherche a encore du mal à fournir de telles pistes d'action tout faisant les mises en garde qui s'imposent et en montrant bien les contraintes et les enjeux conceptuels qui font partie intégrante de la recherche sur le lieu et la santé.

Les articles réunis pour ce numéro spécial donnent un aperçu de la contribution originale des chercheurs canadiens à l'importante question du lieu dans le domaine de la santé des populations. Ils abordent aussi les enjeux que nous venons de mentionner. Les sept articles, tirés d'exposés présentés pendant l'atelier de l'ISPC, s'accompagnent de commentaires de deux responsables de l'élaboration des politiques qui étaient aussi à cet atelier.

Dunn et coll. se penchent sur plusieurs enjeux conceptuels sur la représentation de l'espace dans la recherche sur le lieu et la santé. Ils remettent en question la notion selon laquelle les effets du lieu sont universels et égaux dans leur ampleur, et ils contestent les interprétations fondées sur ces hypothèses. Gauvin et coll. réfléchissent à la définition du " quartier ». Ils font le récit de l'expérience vécue par leur équipe de recherche pour tenter de définir les quartiers malgré le problème endémique des unités spatiales modifiables et des différences énormes entre les structures administratives qui servent à définir les quartiers ou les collectivités à Calgary et à Montréal. Oliver et Hayes analysent les enjeux scalaires de la répartition géographique des disparités dans l'état de santé. Leur comparaison des données d'autoévaluation de la santé tirées de l'Enquête sur la santé dans les collectivités canadiennes, à l'échelle du secteur de recensement et à celle de l'aire de diffusion, met au jour des configurations très différentes. Le secteur de recensement, lorsqu'il est utilisé comme unité d'analyse, masque des écarts dans l'état de santé qui apparaissent beaucoup plus clairement lorsqu'on utilise l'aire de diffusion comme unité.

S'intéressant aux relations sociales et au lieu, Caron et coll. analysent le rôle du soutien social dans l'atténuation des niveaux de détresse psychologique chez les personnes différemment vulnérables aux maladies mentales dans deux quartiers pauvres de Montréal. Ils concluent que le soutien affectif et la présence de personnes perçues comme étant des sources de stress expliquent la plupart des écarts observés dans les niveaux de détresse mesurés. L'étude de De Koninck et Pampalon se distingue des autres articles de ce supplément (et des études publiées sur la question), car on y analyse les perceptions individuelles des rapports qui existent entre le lieu et la santé. En s'interrogeant sur les raisons données par les gens pour expliquer leur choix d'un lieu de résidence, les deux chercheurs nous laissent entrevoir la possibilité d'une relation de réciprocité entre l'ambiance et la composition du quartier. Il y a plusieurs raisons pour lesquelles certaines personnes se regroupent dans certains quartiers, et ces raisons ne sont pas les mêmes pour tous les voisins; la capacité de choisir le lieu où l'on élit domicile est ellemême liée à certains aspects du lieu. Roos et coll., dans leur comparaison entre des frères et sœurs du même sexe vivant dans une même famille et les enfants d'autres familles du même quartier, constatent que les caractéristiques du quartier n'expliquent pas toutes les différences dans les taux d'utilisation des services de santé. Les caractéristiques des ménages sont beaucoup plus importantes pour expliquer ces taux d'utilisation. Lagacé et coll. décrivent les différences dans l'état de santé des populations rurales du Canada et de l'Australie.

Dans son commentaire, Patychuk réfléchit à la valeur informative de chacun des articles de ce numéro et décrit plusieurs projets, en cours dans des villes canadiennes, qui visent à améliorer la santé des populations vivant à tel ou tel endroit. Enfin, Benigeri décrit l'utilisation des systèmes d'information géographique par les autorités sanitaires de Montréal.

Ce numéro spécial et les recherches qu'il renferme n'aurait pas vu le jour sans l'aide de l'Initiative sur la santé de la population canadienne. Au nom de tous nos collaborateurs, je tiens à remercier l'ISPC pour son appui.

\section{BIBLIOGRAPHIE}

1. Potvin L, Avargues MC, Berghmans L, Bilodeau A, Bourdages J, Brunelle Y, Hartz Z. L'intégration de la promotion et de la prévention dans les systèmes de santé. Promotion et Éducation 2005; suppl. 3.

2. Krieger N. Theories for social epidemiology in the 21st century: an ecosocial perspective. Int J Epidemiol 2001;30:668-77.

3. Macintyre S, Ellaway A. Ecological approaches: rediscovering the role of the physical and social environment. Dans : Berkman LF, Kawachi I (éd.), Social Epidemiology. New York, Oxford University Press, 2000; 332-48.

4. Williams GH. The determinants of health: structure, context and agency. Sociol Health Illness 2003;25:131-54.

5. Initiative sur la santé de la population canadienne. Kachimaa Mawiin - Certitudes possibles: Faire une place au lieu dans la recherche et les politiques en matière de santé. Ottawa, Institut canadien d'information sur la santé, 2005. 